\title{
Remote Abstraction of an Installed Dark Fiber Network using Noise to Signal Ratio
}

\author{
David J. Ives, F. J. Vaquero Caballero and Seb J. Savory \\ Electrical Engineering Division, University of Cambridge, 9 JJ Thomson Avenue, Cambridge, CB3 OFA, UK. \\ di231@cam.ac.uk
}

\begin{abstract}
A dark fiber network was partially abstracted utilizing network monitors, prior information and a single probe channel. Validation employed $13 \times 200 \mathrm{~Gb} \cdot \mathrm{s}^{-1}$ DWDM signals transmitted at the optimum launch power, with measured performance $0.2 \mathrm{~dB}$ better than abstracted. OCIS codes: (060.4510) Optical communications, (060.4256) Networks, network optimization.
\end{abstract}

\section{Introduction and Motivation}

The most effective use of network resources requires knowledge of the physical layer impairments such that the best modulation format and FEC coding can be correctly chosen to maximize the error free data rate. Recently a number of authors have used learning algorithms to learn about the physical network and to predict the quality of transmission of future light paths [1-3]. Here we start with an uncommissioned network and systematically probe the properties to create a simple impairment aware abstraction of the underlying physical network that gives the management plane sufficient information to predict performance without requiring complex computation.

Consider an optical network where the links are locally optimized ready for a full DWDM load. These links are abstracted for the global network manager as transport links with length(latency), wavelength resources and quality of transmission degradation expressed as a Noise to Signal Ratio, NSR. The end to end quality of transmission can then be quickly estimated as the sum of NSR along a given route and used to determine the allowed transmission formats.

\section{Remote Probe Methodology}

We wish to abstract the National Dark Fibre Infrastructure (NDFIS), a six node installed fiber test bed linking Cambridge to London, Southampton and Bristol. It consists of dark fiber pairs linking nodes based on Polatis fiber space switches that can be remotely configured to connect between input and output fibers and EDFAs.

At the local node we used a Ciena WaveLogic 3 coherent optical transceiver to provide a $100 \mathrm{~Gb} \cdot \mathrm{s}^{-1}$ probe signal, modulated at $34.5 \mathrm{GBd}$, PM-QPSK and pre-compensated for $30000 \mathrm{ps} \cdot \mathrm{nm}^{-1}$ of chromatic dispersion. The QPSK modulation allowed more sensitive montioring of the received SNR using the moment based calculation [4], while the pre-compensation of chromatic dispersion enhances the nonlinear interference and moves the transmission into a regime where the GN model [5] applies. The probe signal was transmitted from the local node to each remote node in turn and looped back to the local node. To estimate the properties of each span we measure the overall transmission performance using the Ciena WaveLogic 3 as the launch power into the span under consideration was varied while keeping the launch power in all other spans fixed. We also monitor the received optical spectrum with an OSA and the optical power at all the Polatis switch outputs and all the EDFA inputs and outputs. Figure 1(left) shows the sequence of total launch power into each fiber span for the three node, eight span, part of the NDFIS used.

\section{Network Parameter Estimation and Abstraction}

We wish to obtain the following network parameters; span loss, EDFA noise figure, fiber length, fiber attenuation coefficient, fiber chromatic dispersion coefficient and fiber nonlinear coefficient. From these physical parameters we will estimate the optimal launch power for each fiber span and the NSR degradation of each link. We have a number of disparate sources of information including the installation tests, our probe measurements which include the remote optical power monitors, OSA traces from the local node and transmission performance from the Ciena WaveLogic 3.

The fiber span length and attenuation coefficient were obtained from the OTDR fiber installation acceptance tests where an average attenuation coefficient of $0.21 \mathrm{~dB} \cdot \mathrm{km}^{-1}$. The fiber chromatic dispersion was obtained from the fiber installation tests and also the estimated accumulated chromatic dispersion for each loop back from the Ciena WaveLogic 3. The accumulated chromatic dispersion for the installation tests and Ciena Wavelogic 3 agreed within $1 \%$. The fiber type appeared similar with an average chromatic dispersion of $16.4 \mathrm{ps} \cdot \mathrm{nm}^{-1} \cdot \mathrm{km}^{-1}$. The overall fiber span loss was estimated from the ratio of the switch output power to the input power of the following EDFA. The span 
losses estimated from these power monitors were found to be $1 \pm 0.4 \mathrm{~dB}$ higher than the installation test results which is consistent given the expected additional losses of the switch and patch lead connectors.
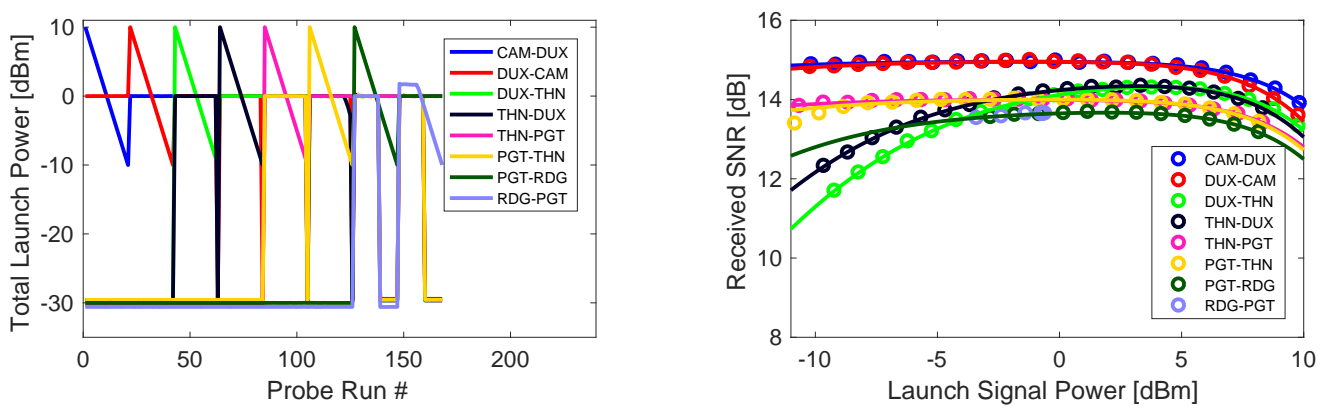

Fig. 1. The total optical launch power for each fiber span as the network is probed(left) and (right) the received symbol SNR vs signal launch power into each fiber, circles measured and lines from a three term fit.

One of the difficulties with remote optical power monitors is that they record the total optical power which includes both signal and accumulated ASE noise. Since the signal bandwidth $<50 \mathrm{GHz}$ while the noise bandwidth $\approx 5000 \mathrm{GHz}$ the ASE noise can have a significant impact on the total optical power. We need to understand the proportions of signal power and ASE noise power at each remote monitor to accurately estimate the signal launch power into each fiber span. At the local node we are able to estimate the ratio of total optical power to signal power using an OSA. This information was supplemented with estimates of the total power and channel power made by the Ciena WaveLogic 3.To estimate the total optical power to signal power ratio at remote locations a simple signal and noise power accumulation model was developed. For each fiber span the signal and noise power were assumed to be equally attenuated. For the EDFAs the following model was used,

$$
S_{\text {out }}=g 10^{\Delta / 10} S_{\text {in }}, \quad N_{\text {out }}=g N_{\text {in }}+10^{N F / 10} h v g B, \quad g=10^{G / 10}\left(\frac{S_{\text {in }}+N_{\text {in }}}{S_{\text {in } 10^{\Delta / 10}+N_{\text {in }}}}\right),
$$

where $S$ is the signal power, $N$ is the noise power, $G$ is the nominal amplifier set gain [dB], $\Delta$ is the gain difference between the signal wavelength and the ASE noise average wavelength $[\mathrm{dB}]$ and $g$ is the linear gain at the ASE noise average wavelength. $N F$ is the amplifier noise figure $[\mathrm{dB}], h$ is Planck's constant, $v$ is the optical carrier frequency and $B$ is the ASE noise bandwidth $5000 \mathrm{GHz}$. The amplifier noise figure was estimated by comparing the noise accumulation model with the measured total optical power to signal power ratios for each loop back and each launch power. Three types of amplifier were present in the experiment a pre-amplifier and booster amplifier each of $20 \mathrm{~dB}$ gain and a general purpose $25 \mathrm{~dB}$ gain amplifier. The least squares error between the accumulation model and measured ratios was minimised by exhaustive search of the three noise figures, each over the range 3 to $6 \mathrm{~dB}$, and the gain difference, $\Delta$ over the range -1 to $0 \mathrm{~dB}$. Noise figures of $4.35,3.70$ and $5.15 \mathrm{~dB}$ along with $\Delta$ of $-0.35 \mathrm{~dB}$ were found to best fit the observed total optical power to signal power ratios.

The EDFA noise figure and gain difference were used to estimate the launch power into each fiber span. Figure 1 shows the symbol SNR measured from the Ciena Wavelogic 3 received constellation as a function of the fiber launch power along with the fitted three parameter nonlinear transmission model [6].The fiber span length, attenuation coefficient and chromatic dispersion were used to calculate the nonlinear interference coefficient based on the GN model. The fiber nonlinear coefficient, $\gamma$, was obtained as a the scaling factor to the NLI coefficient from the three term fit to the measured symbol SNR vs launch power. The nonlinear coefficient was found to be between 1.1 and $1.3 \mathrm{~W}^{-1} \cdot \mathrm{km}^{-1}$.

The estimated transmission parameters were used with the LOGO model [7] to estimate the optimum launch power for each fiber span under a full load of 80 DWDM channels on a $50 \mathrm{GHz}$ grid. From this launch power the NSR degradation due to each link was estimated as shown in the abstraction of figure 2(bottom).

\section{Performance Validation}

To verify the performance of the network against the abstraction, 13 DWDM channels each $200 \mathrm{~Gb} \cdot \mathrm{s}^{-1}, 34.5 \mathrm{GBd}$, PM-16QAM on a $50 \mathrm{GHz}$ grid were launched into the network and looped back at Reading. The performance of the central channel was monitored, the symbol SNR from the moments of the received constellation modified for the transmitted constellation [8] and its pre-FEC BER. Figure 2(top left) shows the arrangement to multiplex the 13 WDM channels. The 12 DFB lasers were bulk modulated by a modified Ciena WaveLogic 3 line card with $820 \mathrm{ps} \cdot \mathrm{nm}^{-1} \mathrm{pre}-$ 

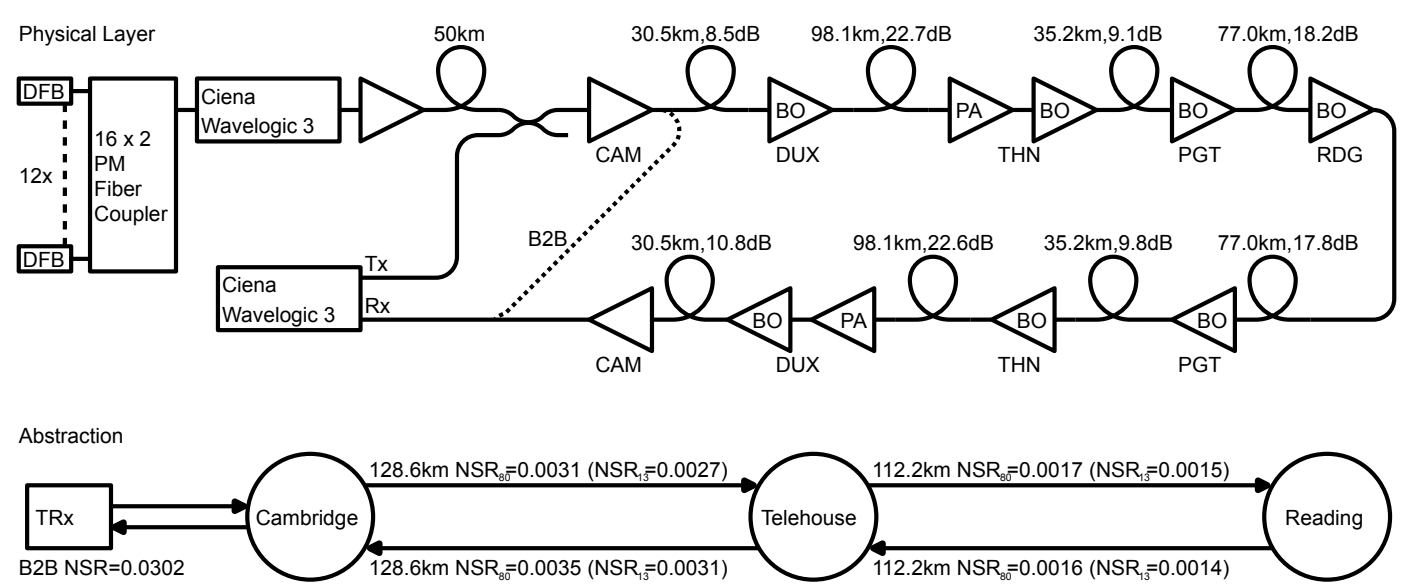

Fig. 2. Experimental layout of the 13 DWDM test source and physical installed network (top) and the abstracted network (bottom). The link NSR figures are for 80 and 13 DWDM channels under the incoherent GN model approximation.

compensation before being passed through $50 \mathrm{~km}$ of fiber to decorrelate the signals. The 12 bulk modulated signals were combined with the central channel using a passive coupler before being amplified for the first fiber span.

The back to back NSR of the central channel through the coupler and launch EDFA was found to be $30.2 \times 10^{-3}$. From the abstracted NSR degradation for each link we expect the loop back route to Reading combined with the back to back NSR, under full load of 80 channels, to have an overall NSR of $40.1 \times 10^{-3}$, and for 13 channels an NSR of $38.9 \times 10^{-3}$. This is less than the theoretical maximum NSR for PM-16QAM with a pre-FEC BER of $<3 \%$ of $65.3 \times 10^{-3}$. The measured NSR was $38.0 \times 10^{-3}$ for the 13 channel load, slightly better than expectation. The preFEC BER of the central channel was $\approx 1 \%$ at the nominal optimal launch power and was below $3 \%$ for launch power deviations of $\pm 6 \mathrm{~dB}$. This compares well with an expected launch power variation range of $-7 \mathrm{~dB}$ to $4 \mathrm{~dB}$ for a fully loaded network.

\section{Conclusions}

We have abstracted an installed fiber network by remotely probing the transmission characteristics and combining this with other available information sources. The accuracy of the abstraction was confirmed with the transmission of a $200 \mathrm{~Gb} \cdot \mathrm{s}^{-1} \mathrm{PM}-16 \mathrm{QAM}$ signal surrounded by 12 similar aggressor channels. A received NSR 5\% lower, equivalent to an SNR only $0.2 \mathrm{~dB}$ higher, than that predicted from the abstracted fully loaded DWDM network was achieved. Under the actual load conditions the measured SNR was only $0.1 \mathrm{~dB}$ higher than expectation, this final difference is fully explained with the more complex EGN model of NLI. Future work will look at other probing strategies and will also consider more formal methods to accurately combine information from multiple disparate sources.

\section{Acknowledgments}

The authors acknowledge funding support from the UK EPSRC (through the project INSIGHT EP/L026155/2) and from Ciena and acknowledge use of the EPSRC National Dark Fibre Infrastructure Service, NS/A000021/1.

\section{References}

1. R. Borkowski, et al., "Robust cognitive-GN BER estimator for dynamic WDM networks,” in "Proc. ECOC 2014,” P.2.13.

2. M. Bouda, et al., "Accurate Prediction of Quality of Transmission with Dynamically Configurable Optical Impairment Model," in "Proc. OFC," (Anaheim, CA. (USA), 2017), Th1J.4.

3. F. Meng, et al., "Robust Self-Learning Physical Layer Abstraction Utilizing Optical Performance Monitoring and Markov Chain Monte Carlo," in "Proc. ECOC 2017," (Göteburg, (SE), 2017), W.3.A.2.

4. D. J. Ives, et al., "Estimating OSNR of equalised QPSK signals," Opt. Express 19, B661-B666 (2011).

5. P. Poggiolini, et al., "The GN-Model of Fiber Non-Linear Propagation and its Applications," J. Lightw. Technol. 32, 694-721 (2014).

6. D. J. Ives, et al., "Single Channel Probe Utilizing the EGN Model to Estimate Link Parameters for Network Abstraction," in "Proc. ECOC 2017," (Göteborg, (SE), 2017), P2.SC6.13.

7. P. Poggiolini, et al., "The LOGON Strategy for Low-Complexity Control Plane Implementation in New-Generation Flexible Networks," in "Proc. OFC 2013," (Anaheim, CA. (USA), 2013), OW1H.3.

8. M. S. Faruk, et al., "In-Band Estimation of Optical Signal-to-Noise Ratio From Equalized Signals in Digital Coherent Receivers," IEEE Photonics J. 6, 1-9 (2014). 